\title{
Sex-specific correlation of IGFBP-2 and IGFBP-3 with vitamin D status in adults with obesity: a cross-sectional serum proteomics study
}

Nasser M. Al-Daghri ${ }^{1,2}$, Antigoni Manousopoulou ${ }^{3}$, Majed S. Alokail ${ }^{1,2}$, Sobhy Yakout ${ }^{1,2}$, Amal Alenad ${ }^{1,2}$, Diana J. Garay-Baquero ${ }^{3}$, Miltiadis Fotopoulos ${ }^{3}$, Jie Teng ${ }^{3,4}$, Omar Al-Attas ${ }^{2}$, Yousef Al-Saleh², Shaun Sabico ${ }^{1,2}$, George P. Chrousos ${ }^{5}$ and Spiros D. Garbis (1) 3,6,7

\begin{abstract}
Objective: Subjects with low vitamin D levels are at risk of cardiometabolic disease. The aim of this study was to identify novel serological markers linking vitamin D status with cardiometabolic profile in non-diabetic adults with obesity.

Methods: For the discovery phase, we used quantitative serum proteomics in sex-matched, age-matched and BMlmatched subjects with obesity [BMl: $\left.25-35 \mathrm{~kg} / \mathrm{m}^{2}\right]$ and low $[25(\mathrm{OH}) \mathrm{D}<50 \mathrm{nmol} / \mathrm{L}]$ vs. high vitamin $\mathrm{D}$ status $[25(\mathrm{OH}) \mathrm{D}>50$ $\mathrm{nmol} / \mathrm{L}](n=16)$. For the validation phase, we performed ELISA in a larger cohort with similar characteristics $(n=179)$.

Results: We identified 423 and 549 differentially expressed proteins in the high vs. low vitamin D groups of the male and female cohorts, respectively. The small molecule biochemistry protein networks and the glycolysis $\mid$ gluconeogenesis pathway were significantly enriched in the DEPs of both sexes. As surrogate markers to these processes, the insulin-like growth factor binding protein -2 (IGFBP-2) was upregulated in males, whereas IGFBP-3 was upregulated in females from the high Vitamin D status. This sex-specific trend was confirmed using Luminex ELISA to an independent but clinically analogous cohort of males $(n=84, p=0.002)$ and females $(n=95, p=0.03)$.

Conclusions: The high Vitamin D status correlated with the serological upregulation of IGFBP-2 in males and IGFBP-3 in females with obesity and may constitute surrogate markers of risk reduction of cardiometabolic disease.
\end{abstract}

\section{Introduction}

Vitamin D is an ancient hormone, originally produced by archaebacteria, phytoplankton and zooplankton dating

Correspondence: Spiros D. Garbis (sgarbis@caltech.edu)

${ }^{1}$ Biochemistry Department, College of Science, Biomarkers Research Program, King Saud University, Riyadh, Saudi Arabia

${ }^{2}$ Biochemistry Department, Prince Mutaib Chair for Biomarkers of Osteoporosis, King Saud University, Riyadh, Saudi Arabia

Full list of author information is available at the end of the article.

These authors are contributed equally: Nasser M. Al-Daghri,

Antigoni Manousopoulou back to over 500 million years ${ }^{1}$. Almost all mammalian cell types express the vitamin $\mathrm{D}$ receptor, suggesting that vitamin $\mathrm{D}$ may exhibit a pleiotropic effect in addition to its well-established role in calcium and phosphorus homoeostasis $^{2}$. A clinically relevant marker used to measure vitamin D status is circulating levels of $25(\mathrm{OH})$ $\mathrm{D}^{3}$. Although there is significant controversy in this field, vitamin $\mathrm{D}$ insufficiency is defined by the Institute of Medicine (IOM) as serum 25(OH)D levels lower than 50

\section{(c) The Author(s) 2018}

(c) Open Access This article is licensed under a Creative Commons Attribution 4.0 International License, which permits use, sharing, adaptation, distribution and reproduction cc) in any medium or format, as long as you give appropriate credit to the original author(s) and the source, provide a link to the Creative Commons license, and indicate if changes were made. The images or other third party material in this article are included in the article's Creative Commons license, unless indicated otherwise in a credit line to the material. If material is not included in the article's Creative Commons license and your intended use is not permitted by statutory regulation or exceeds the permitted use, you will need to obtain permission directly from the copyright holder. To view a copy of this license, visit http://creativecommons.org/licenses/by/4.0/. 
$\mathrm{nmol} / \mathrm{L}^{4}$. Notably, the Endocrine Society suggested that circulating 25(OH)D should be maintained at higher concentrations ( 75 to $80 \mathrm{nmol} / \mathrm{L}$ ) for extra-skeletal health benefits ${ }^{5}$ with no known toxicity at this level ${ }^{6,7}$. Across countries in all continents, the mean serum concentration of $25(\mathrm{OH}) \mathrm{D}$ is around $50 \mathrm{nmol} / \mathrm{L}$, suggesting that approximately $50 \%$ of these populations have vitamin D insufficiency ${ }^{8,9}$.

Emerging evidence suggests the extra-skeletal, pluripotent effects of vitamin D in reducing the risk of adverse cardiometabolic outcomes. A meta-analysis, including 65,000 prospectively monitored participants, showed that the group with the lowest compared to the highest serum 25(OH)D levels had a relative risk of 1.5 (1.3 to 1.8$)$ for total incidents of cardiovascular disease and 1.6 (1.3 to 2.1) for stroke $e^{10}$. Another meta-analysis of approximately 500,000 participants found an inverse association with allcause mortality for circulating 25(OH)D levels up to $75 \mathrm{nmol} / \mathrm{L}^{11}$. An additional dose-response meta-analysis from 34 studies involving 180,667 participants demonstrated that serum $25(\mathrm{OH}) \mathrm{D}$ levels were inversely correlated with total number of CVD events and CVD mortality ${ }^{12}$. Decreased 25(OH)D levels may affect cardiovascular risk either directly, for example by increasing blood pressure through the renin-angiotensin system, or indirectly, by influencing inflammation, myocardial function, vascular calcification and parathyroid hormone levels ${ }^{13}$.

An additional potential extra-skeletal effect of vitamin D is its chemopreventive effects against type II diabetes mellitus (T2DM). The meta-analysis of well-powered clinical studies have shown that subjects with low 25(OH) $\mathrm{D}$ levels have an increased risk of developing T2DM compared to those with a normal vitamin D status ${ }^{14}$. Results from a population study in Victoria, Australia showed an inverse association between vitamin D status and risk factors of T2DM, including fasting plasma glucose and HbA1c levels ${ }^{15}$. A recent systematic review and meta-analysis that included almost 30,000 subjects, found that lower $25(\mathrm{OH}) \mathrm{D}$ levels were significantly associated with an increased risk of developing diabetes among older adults $^{16}$. Low vitamin D status may lead to insulin resistance by impairing insulin secretion and compromising pancreatic beta-cell function, all hallmark features of $\mathrm{T}_{2} \mathrm{DM}^{17,18}$. Recent findings suggest that supplementation with vitamin D could positively affect insulin secretion and glucose homoeostasis ${ }^{19,20}$. An additional recently published Mendelian randomisation study in European and Chinese adults provided first ever evidence for a causal effect of higher $25(\mathrm{OH}) \mathrm{D}$ serum levels for the prevention of $\mathrm{T}_{2} \mathrm{DM}^{21}$. As a corollary to these observations, the chemoprevention of T2D prior to its clinical diagnosis in individuals with obesity by means of Vitamin D status correction may also reduce the risk of cardiometabolic disease. However, surrogate protein markers that can easily be measured in serum to gauge such a risk reduction due to Vitamin D status improvement are currently lacking.

The aim of the present study was to perform quantitative serum proteomics in a cross-sectional cohort of nondiabetic adults with obesity and low vs. high vitamin D status in order to identify novel serological markers linking vitamin D status to cardiometabolic disease risk. The relevant differentially expressed proteins chosen as candidate markers were further verified against a larger cross-sectional cohort with the same inclusion/exclusion

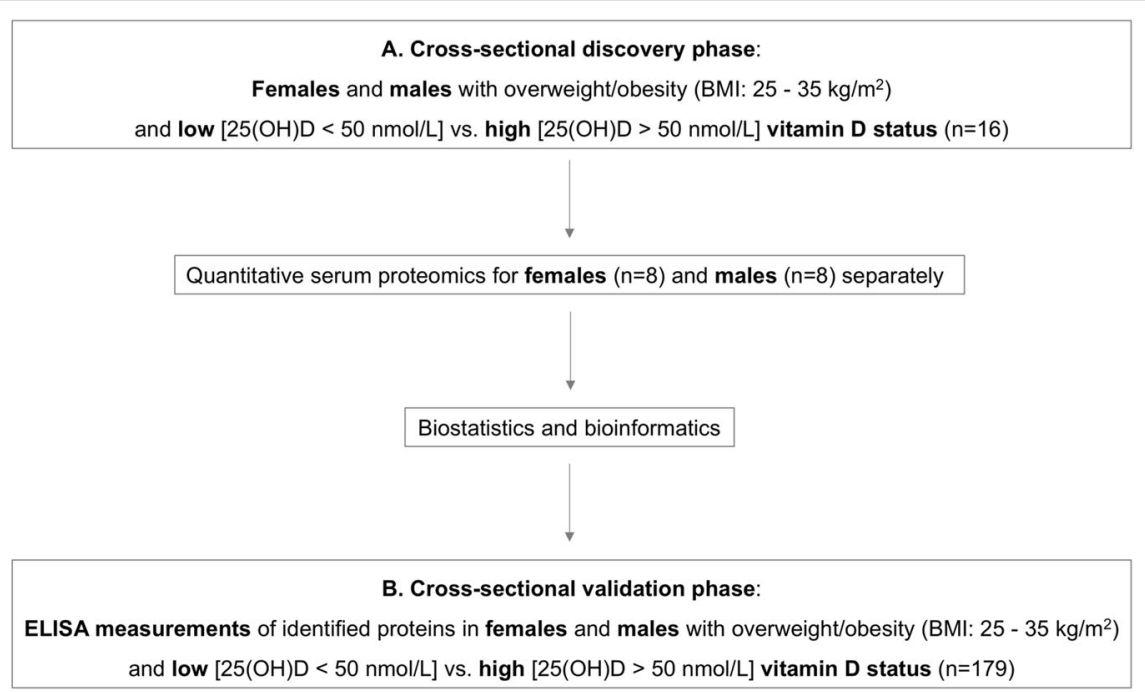

Fig. 1 Study design: a cross-sectional quantitative serum proteomics discovery phase with an ELISA validation phase for the identification of novel serological markers of vitamin D status linked to cardiometabolic outcomes among adults with overweight/obesity 
criteria as for the discovery phase. An overview of the study design is presented in Fig. 1.

\section{Materials and methods \\ Discovery phase: serum proteomics in cross-sectional samples}

The study received ethics approval by the King Saud University Ethics Committee. Reporting of the crosssectional study conforms to the STROBE statement and the broader EQUATOR guidelines ${ }^{22}$. For the crosssectional serum proteomics study, participants were randomly selected from an existing cohort, the Riyadh cohort $2^{23}$. Written informed consent was obtained from all participants. Adults with overweight/obesity (BMI between 25 and $35 \mathrm{~kg} / \mathrm{m}^{2}$ ) and with normal fasting plasma glucose levels (3.9 to $5.5 \mathrm{mmol} / \mathrm{L}$ ) and serum $25(\mathrm{OH}) \mathrm{D}<$ $50 \mathrm{nmol} / \mathrm{L}$ or $>50 \mathrm{nmol} / \mathrm{L}$ were included in the study. Subjects pregnant or breastfeeding, with morbid obesity $\left(\mathrm{BMI}>35 \mathrm{~kg} / \mathrm{m}^{2}\right)$, diagnosed with type 1 or type 2 diabetes mellitus, or with non-alcoholic fatty liver disease were excluded from the study. An abdominal ultrasound was performed to exclude non-alcoholic fatty liver disease among the study participants. Anthropometry and morning blood withdrawal was performed after overnight fasting. Blood collection for all participants took place during the winter months (December to February). The discovery cohort comprised 16 participants (four males with serum $25(\mathrm{OH}) \mathrm{D}<50 \mathrm{nmol} / \mathrm{L}$; four males with serum $25(\mathrm{OH}) \mathrm{D}>80 \mathrm{nmol} / \mathrm{L}$; four females with serum $25(\mathrm{OH}) \mathrm{D}$ $<50 \mathrm{nmol} / \mathrm{L}$; four females with serum $25(\mathrm{OH}) \mathrm{D}>80$ $\mathrm{nmol} / \mathrm{L})$. Participants were asked about their sun exposure habits using a questionnaire as reported before ${ }^{24}$. Total energy and micronutrient (vitamin D, calcium and EPA/DHA) intake was estimated using food frequency questionnaires as reported before ${ }^{24}$. Physical activity levels of the participants were assessed using the WHO Global Physical Activity Questionnaire, as reported previously $^{24}$.

\section{Serum procurement and proteomic analysis}

The procurement and handling of sera was in accordance with the recommendations of the Standard Operating Procedure Integration Working Group (SOPIWG) as adopted by the author's method ${ }^{25}$. Two eight-plex serum proteomics experiments were performed for male and female subjects separately, as we have shown previously that vitamin $\mathrm{D}$ has sex-specific non-skeletal cardiovascular effects ${ }^{24}$. The serum specimens were freshly thawed and vortexed for $2 \mathrm{~min}$. For each participant, $100 \mu \mathrm{L}$ of unprocessed serum were immediately mixed with $400 \mu \mathrm{L} 6 \mathrm{M}$ Guanidine Hydrochloride and subjected to global quantitative serum proteomic analysis using our previously published method ${ }^{24,25}$. In summary, highperformance Size Exclusion Chromatography using three serially connected Waters KW-804 columns at $0.75 \mathrm{ml} / \mathrm{min}$ flow rate and $30{ }^{\circ} \mathrm{C}$ was used to separate the proteins based on their molecular weight differences. The separated low-molecular weight protein segments (molecular weight cutoff $3 \mathrm{kDa}$ ) were dialysis purified and lyophilised to dryness at $4{ }^{\circ} \mathrm{C}$. One-hundred $\mu \mathrm{g}$ of protein from each sample was subjected to trypsin proteolysis and the peptides were chemically labelled using the eight-plex isobaric Tag for Relative and Absolute Quantitation (iTRAQ) reagents, pooled, and offline fractionated with $\mathrm{C}_{4}$ reverse phase chromatography at $\mathrm{pH} 8.0(2.1 \mathrm{~mm} \mathrm{X}$ $150 \mathrm{~mm}, 3 \mu \mathrm{m}$ particle, $120 \AA$ pore, Kromasil, Germany). As a multiplex proteomics workflow, samples were analysed under the same exact experimental conditions. Each fraction was analysed using ultra-high performance $C_{18}$ nano-liquid chromatography at pH $3.5(75 \mu \mathrm{m}$ ID x $50 \mathrm{~cm}$, $2 \mu \mathrm{m}$ particle, $100 \AA$ pore, Hypersil, USA) hyphenated with high-resolution tandem mass spectrometry using the FT-Orbitrap Elite platform.

Unprocessed raw files were submitted to Proteome Discoverer 1.4 for target decoy search against the UniProtKB Homo Sapiens database (release date 10-Jan2015) using SequestHT. Only reporter ion ratios from unique peptides were considered for the quantitation of the respective protein. Median normalisation and $\log _{2}$ transformation was performed for the reporter ion quantification ratios. A protein was considered differentially expressed between the high vs. low vitamin D status when its one-sample, two-tailed, $T$-test $p$-value was $\leq 0.05$ and the mean iTRAQ $\log _{2}$ ratio of high vs. low vitamin D status was higher than \pm 0.3 . All mass spectrometry proteomics data have been deposited to the ProteomeXchange Consortium via the PRIDE partner repository with the dataset identifier PXD009606.

\section{Principal component analysis and bioinformatics with biostatistics informed selection of candidate verification markers}

Principal component analysis using the reporter ion ratios of the all analysed proteins in the male and female cohorts, respectively was performed using ClustVis (https://biit.cs.ut.ee/clustvis/). DAVID (https://david. ncifcrf.gov/) and Ingenuity Pathway Analysis (IPA) (Qiagen, Hilden, Germany) software tools were used to identify canonical pathways and protein networks significantly over-represented in the differentially expressed proteins between high and low vitamin $D$ status groups of the male and female cohorts. Significance was set at $p$-value $\leq 0.05$. Surrogate markers to the identified enriched pathways were further evaluated as candidate verification markers with bibliographic research. As an additional qualifier, retrospective statistical power analysis using the function pwr.2p.test() available within $\mathrm{R}$ (https://www.R-project. $\mathrm{org} /$ ) was applied to the suitable candidate marker as 
reported $^{26}$. The minimum statistical power threshold was set at 0.8 , which factored in the $p$-value, variation, differential expression ratio, and the number of replicate biological observations for the chosen protein analysed from the discovery experiment.

\section{Verification phase: ELISA measurements in an independent cross-sectional cohort}

ELISA measurements of selected proteins identified at the discovery phase were validated in a larger crosssectional cohort of adults with obesity randomly selected from the Riyadh cohort $2^{23}$, using the same inclusion and exclusion criteria as described above for the discovery phase. In total, 179 adults were included, of which 84 males $[n=40$ with $25(\mathrm{OH})<50 \mathrm{nmol} / \mathrm{L} ; n=44$ with 25 $(\mathrm{OH})>50 \mathrm{nmol} / \mathrm{L}]$ and 95 females $[n=47$ with $25(\mathrm{OH})<$ $50 \mathrm{nmol} / \mathrm{L} ; n=48$ with $25(\mathrm{OH})>50 \mathrm{nmol} / \mathrm{L}]$. The size of the verification cohort was based on the logistic regression models requiring a minimum of 10 events per predictor variable ${ }^{27}$, which in our study included sex and 25 $(\mathrm{OH}) \mathrm{D}$ levels. All other clinical parameters listed in Table 2 were not considered, as they remained constant between the cohorts examined.

\section{Insulin-like growth factor binding protein IGFBP-2 and IGFBP-3 ELISA measurements}

IGFBP-2 and IGFBP-3 were targeted for measurement using the commercially available Luminex ELISA kit (Catalog number: HIGFBMAG-53K; Millipore, Billerica, MA, USA) according to manufacturer's instructions. The captured bead complexes were measured with the FLEXMAP 3D system (Luminex Corporation, Austin, TX). The raw data (mean fluorescence intensity) were collected and further processed for calculating protein concentration. The intra-assay and inter-assay coefficient of variation $(\mathrm{CV})$ was $<10$ and $<15 \%$, respectively.

\section{Biochemical analyses}

Fasting glucose levels were measured using an enzymatic assay (hexokinase coupled with glucose 6phosphate dehydrogenase) with a chemical analyser (Konelab, Espoo, Finland). The inter-assay CV was $2.2 \%$. Serum 25(OH)D levels were measured by a specific enzyme-linked immunosorbent assay (IDS, Tyne and Wear, UK). The inter- and intra-assay variability of this assay was 5.1 and $4.6 \%$, respectively.

\section{Clinical data analysis}

Clinical data were analysed using SPSS (Version 25). An unpaired, two-tailed Student $T$-test was applied to compare the clinical and lifestyle characteristics of the low vs. high vitamin D status groups of males and females in the discovery and validation cohorts. A Mann-Whitney $U$ Test was used to compare IGFBP-2 and IGFBP-3 levels in low vs. high vitamin D status groups of males and females in the validation cohort. Parameters are presented as mean \pm standard deviation or median (25th to 75th percentile). A $p$-value less than 0.05 was considered significant.

\section{Results}

The anthropometric, lifestyle and clinical characteristics of the participants in the discovery phase are presented in Table 1 . The low and high vitamin D status groups of the male and female participants were similar with regards to age, BMI, fasting glucose, sun exposure, physical activity and total energy, DHA/EPA and calcium intake. The two groups had significantly different serum $25(\mathrm{OH}) \mathrm{D}$ levels $(p<0.0001)$ as per the inclusion criteria and vitamin D intake $(p<0.0001)$.

In the male and female cohorts of the cross-sectional study, 1297 and 1114 protein groups were profiled, respectively (peptide level FDR $p<0.05$ ). Principal component analysis using the iTRAQ $\log _{2}$ ratio of all analysed proteins in high vs. low vitamin D status groups for male and female subjects of the cross-sectional study is presented in Fig. 2. Of the quantitatively analysed serum proteins, 423 and 549 proteins were differentially expressed in the high vs. low vitamin D status conditions of the male and female cohorts, respectively (Supplementary Tables 1 and 2). IPA analysis showed that protein networks related to small molecule biochemistry were enriched in both male and female cohorts (score = 17 and 19 for males and females, respectively) (Fig. 3a, b). KEGG canonical pathway analysis showed that glycolysis| gluconeogenesis was significantly enriched in the differentially expressed proteins (DEPs) of the male and female cohorts (Fisher exact $p=0.002$ and 0.028 , respectively for males and females) (Fig. 3c). The secreted IGFBP2 and IGFBP3 proteins, as surrogate markers of glucose and fatty acid homeostasis through their engagement with the IGF/Insulin complex ${ }^{55}$, were found to be differentially expressed between low and high Vitamin D status of both male and female cohorts.

In particular, the discovery serum proteomics analysis showed that IGFBP-2 was over-expressed in men with high compared to low vitamin D status [Males: IGFBP2 mean iTRAQ $\log _{2}$ ratio in high vs. low vitamin D status $(\mathrm{SD})=0.6(0.8), p=0.02]$ whereas IGFBP-3 was expressed at higher levels in women with high compared to low vitamin D status [Females: IGFBP-3 mean iTRAQ $\log _{2}$ ratio in high vs. low vitamin $\mathrm{D}$ status $(\mathrm{SD})=0.5(0.5)$, $p=0.001$ ] (Fig. 4a). This sex-specific correlation of vitamin D status with IGFBP-2 and IGFBP-3 was further examined using Luminex ELISA analysis against an independent cross-sectional cohort.

The clinical characteristics of the validation cohort are presented in Table 2. As for the discovery cohort, the low 


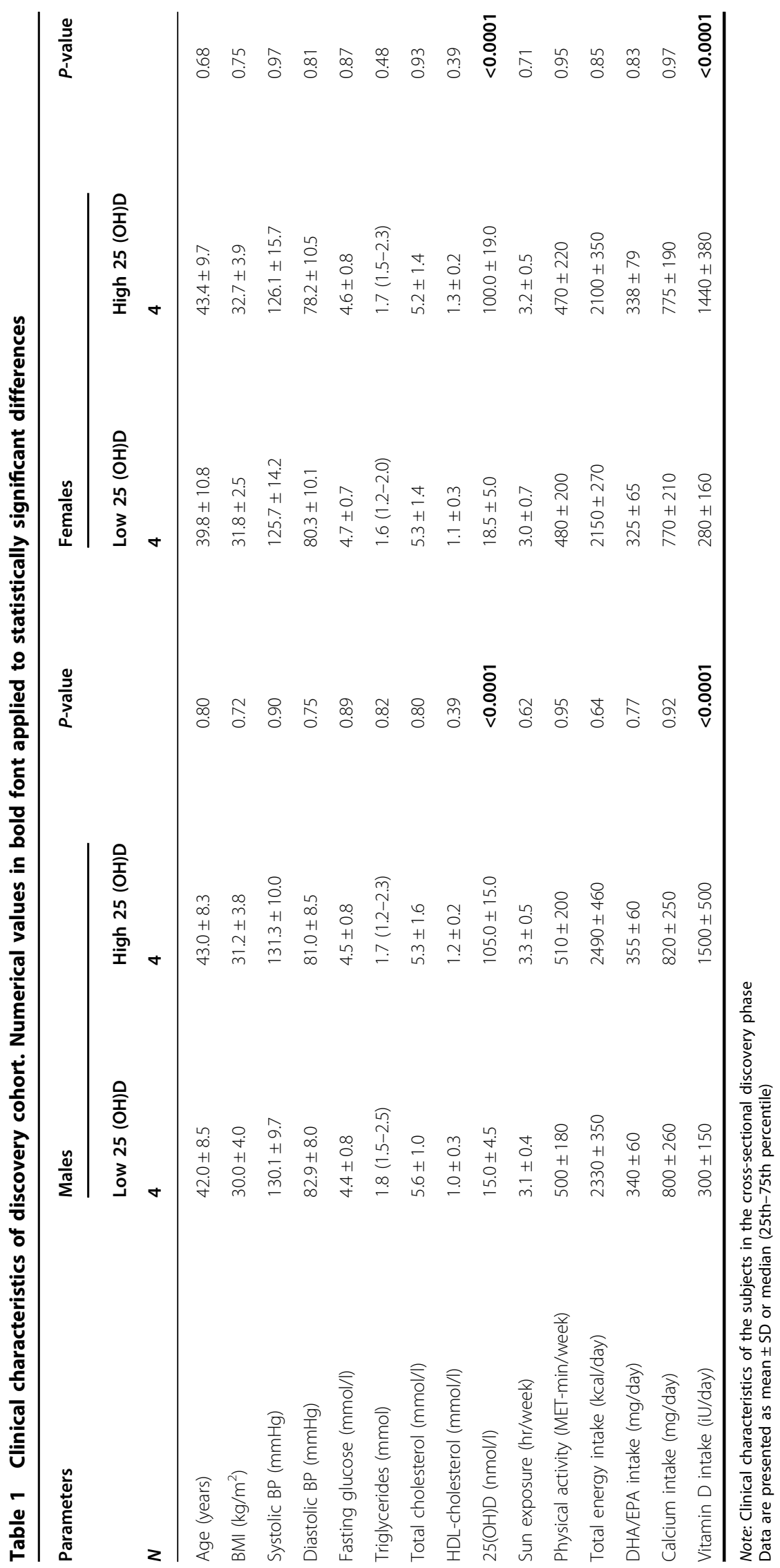




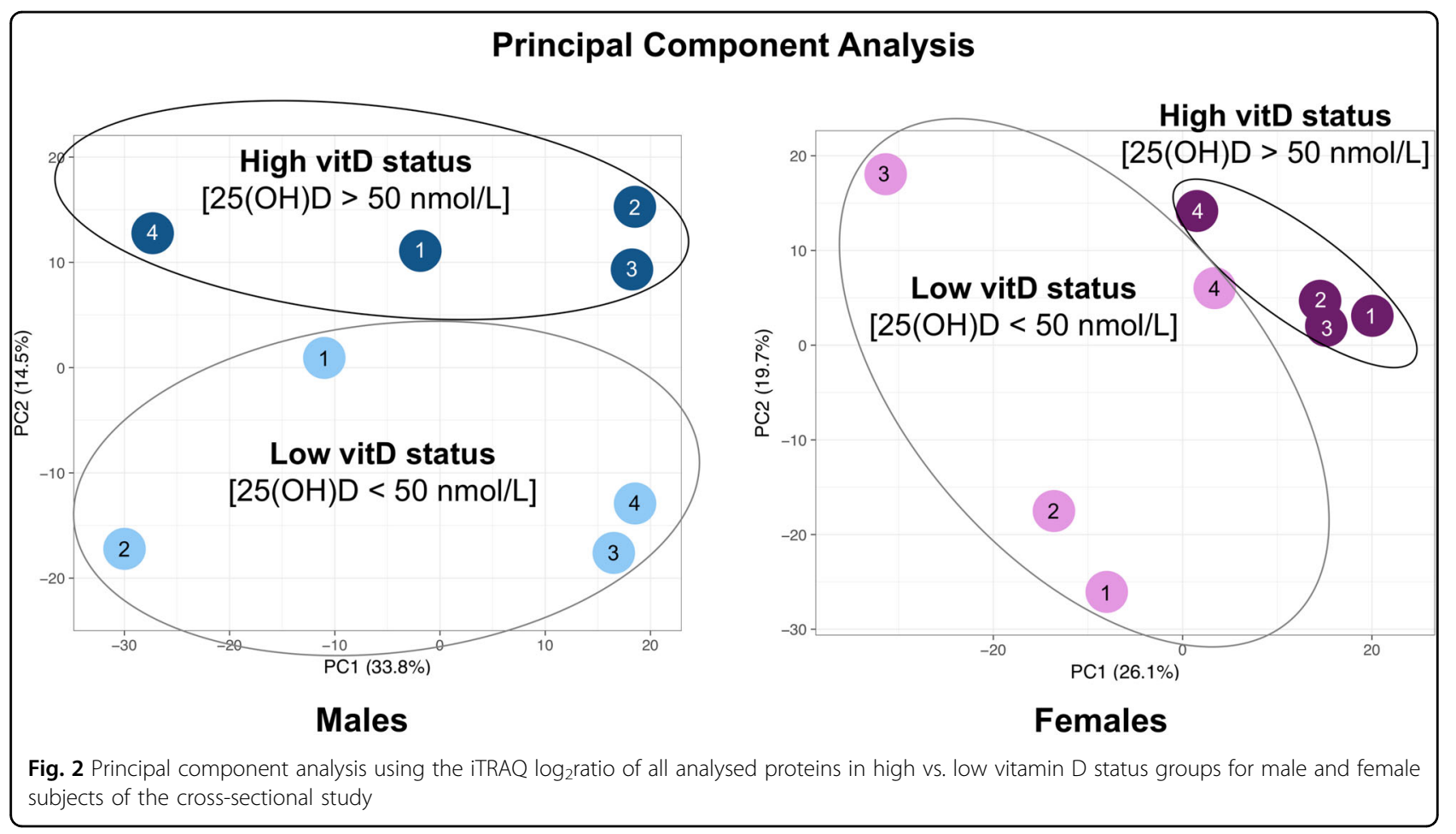

and high vitamin D status groups of the male and female participants were similar with regards to age, BMI, fasting glucose, sun exposure, physical activity and total energy, DHA/EPA and calcium intake. The two groups had significantly different vitamin D intake $(p<0.0001)$ and vitamin D status $(p<0.0001)$. ELISA measurements confirmed the sexually dimorphic correlation of IGFBP-2 and IGFBP-3 with vitamin D status among adults with obesity [Males: IGFBP-2 Low vitamin D status median (25th to 75th percentile $)=5.1 \quad(2.9-11.1)$, High vitamin $\mathrm{D}$ status median (25th to 75 th percentile) $=10.0(5.6-22.1)$, $p=0.002$; IGFBP-3 Low vitamin D status median (25th to 75 th percentile $)=5.4(3.4-5.6)$, High vitamin D status median (25th to 75th percentile) $=5.5(3.9-5.8), p=0.66$ ] [Females: IGFBP-2 Low vitamin D status median (25th to 75th percentile $)=11.4 \quad(6.6-23.4)$, High vitamin D status median (25th to 75 th percentile) $=13.4(5.7-25.8)$, $p=0.99$; IGFBP-3 Low vitamin D status median (25th to 75th percentile $)=2.4(1.6-2.8)$, High vitamin D status median (25th to 75th percentile) $=2.8(2.7-4.5), p=0.03$ ] (Fig. 4b).

\section{Discussion}

Individuals with obesity and low Vitamin D status exhibit a substantially increased risk of cardiometabolic disease including its key component, T2 $\mathrm{D}^{2,10-21}$. Such an increased risk is manifested well before the clinical diagnosis of cardiometabolic disease. Identifying novel protein markers at the minimally invasive blood serum level may improve our understanding and aggressive management of such at risk individuals. In this capacity, such serological protein markers may guage the effectiveness of Vitamin D intervention on reducing risk of cardiometabolic disease to individuals with obesity and low Vitamin D status. To address this need, the main aim of this study was to identify novel serum proteins as surrogate markers that may correlate Vitamin D status with cardiometabolic disease risk in non-diabetic adults with obesity using an agnostic quantitative serum proteomics approach (Fig. 1) based on the method developed by the authors ${ }^{24,25}$. The Principle Component Analysis of the entire serum proteome observed (Fig. 2) captured the anticipated sexual dimorphic protein expression, as previouly reported ${ }^{24,25}$. Futher bioinformatics interpretation (using Ingenuity and KEGG Pathway Analysis) of the differentially expressed proteins $(p<0.05)$ between the high vs.low Vitamin D status conditions of the male and female cohorts (Suppl. Tables 1 and 2) resulted in the sexual dimorphic enrichment of the small molecule biochemistry protein networks (male and female enrichment scores of 17 and 19, respectively, Fig. $3 \mathrm{a}$ and b) and the glycolysis $\mid$ gluconeogenesis pathway (Fisher exact $p=0.02$ for males and $p=0.028$ for females, Fig. 3c). Interestingly, and consequent to the serum proteomic method used in this study, key participatory proteins observed in these enriched metabolic processes were of exosomal origin, as listed in the highly curated ExoCarta database (http:// www.exocarta.org). Notable exosomal derived proteins 


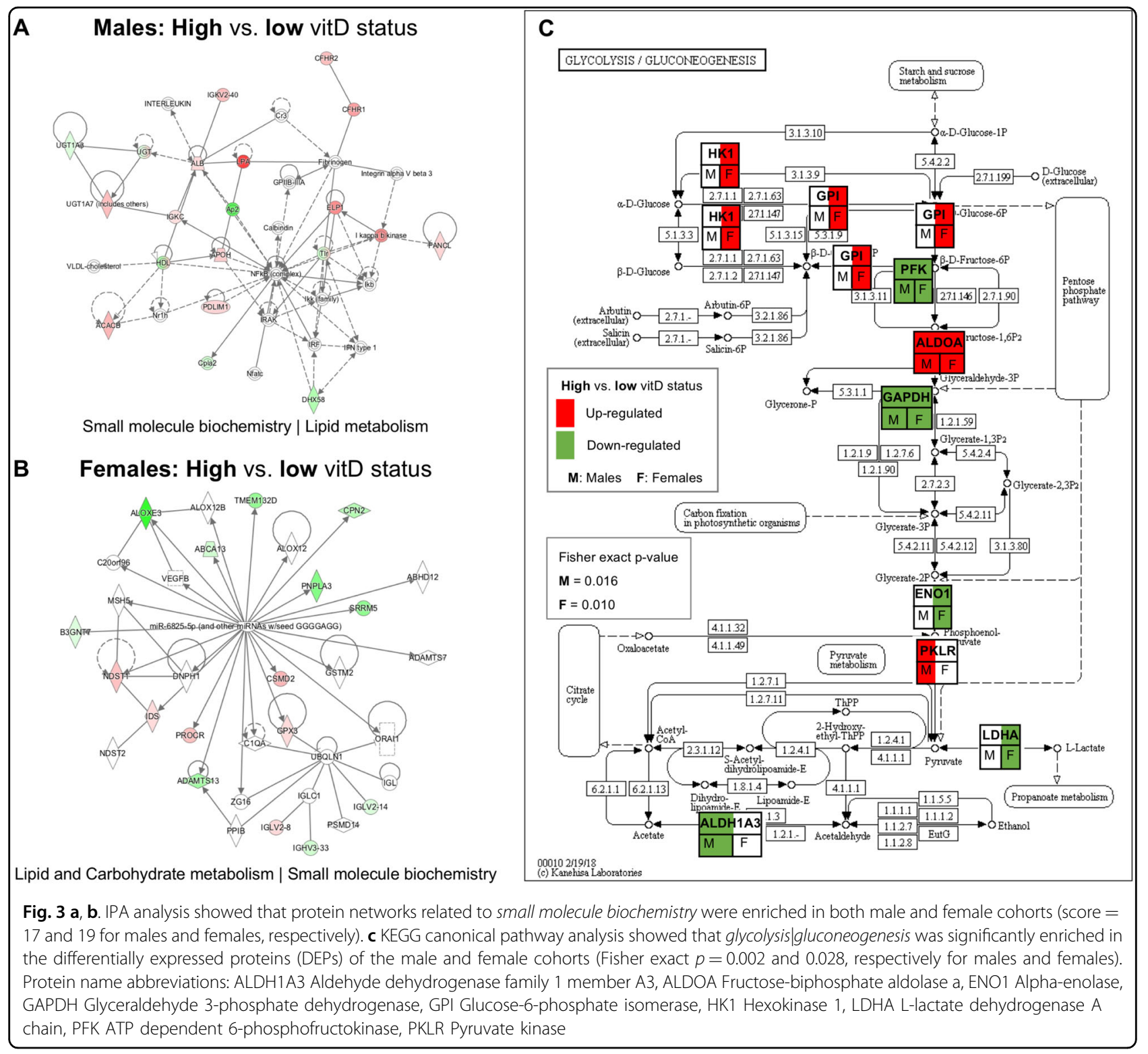

found to be differentially exprerssed between the male high vs. low Vitamin D status conditions in the small molecule biochemistry network were the PDZ and LIM domain 1 (PDLIM1); acetyl-CoA carboxylase beta $(A C A C B)$; and UDP glycosyltransferace 1 family, polypeptide A8 (UGT1A8) (Fig. 3a), whereas the differentially expressed proteins found in the female high vs. low Vitamin D status condition of the same network were carboxypeptidase $\mathrm{N}$, polypeptide 2 (CPN2), protein $\mathrm{C}$ receptor, endothelial (PROCR); and ADAM metallopeptidase with thrombospondin type 1 motif, 13 (ADAMTS13) (Fig. 3b). For the glycolysis|gluconeogenesis pathway, key exosomal derived proteins differentially expressed in the high vs. low Vitamin D status included aldolase $\mathrm{A}$, fructose-bisphosphate $(A L D O A)$ found to be upregulated in both sexes; pyruvate kinase liver and red blood cell $(P K L R)$ found upregulated in males; enolase 1, alpha (ENO1) found downregulated in females; and hexokinase 1 (HK1) found upregulated in females (Fig. $3 c)$. An overarching regulatory process controlling the above described sugar and fatty acid metabolic traits is the growth hormone--insulin-like growth factor axis ${ }^{28,29}$. More precisely, the growth hormone-insulin-like growth factor axis is an evolutionary conserved system that controls somatic growth and metabolism ${ }^{28}$. Growth hormone $(\mathrm{GH})$, a peptide hormone secreted by the anterior pituitary gland, is a stress hormone that counteracts the action of insulin and directly increases the concentration of glucose in the blood ${ }^{29}$. GH administration has been shown to increase gluconeogenesis and glycogenolysis 


\section{A} Serum proteomics

IGFBP2

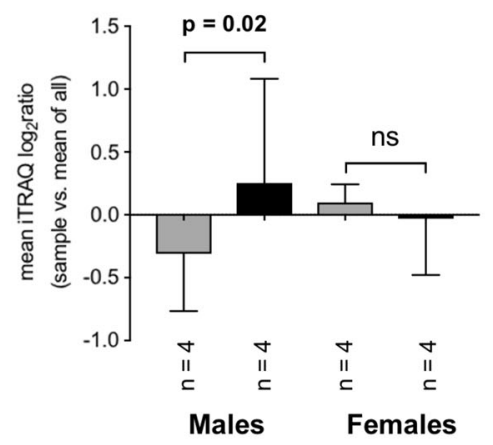

\section{IGFBP3}

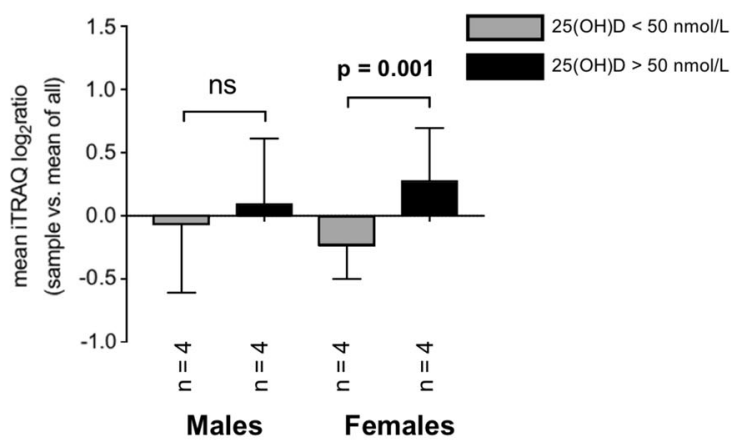

B

ELISA measurements

IGFBP2

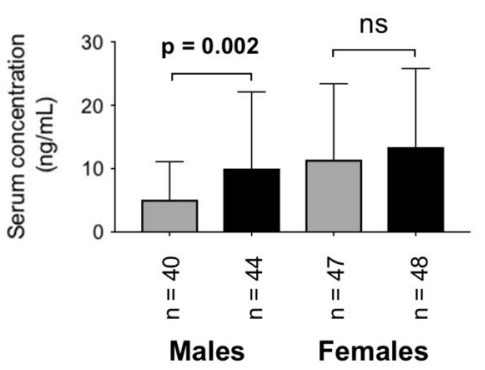

IGFBP3

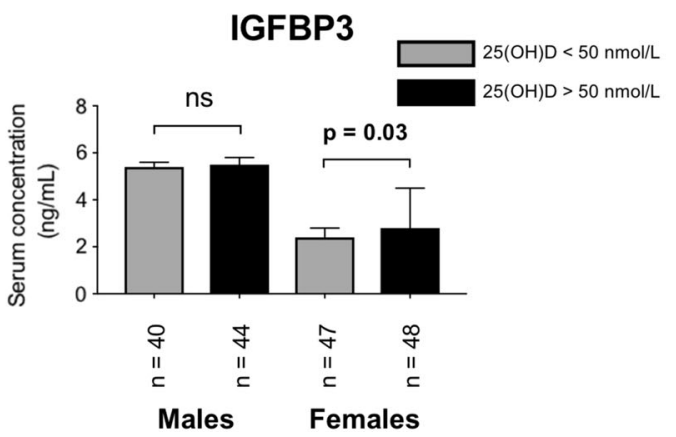

Fig. 4 a Quantitative proteomic analysis of IGFBP-2 and IGFBP-3 in the male and female cross-sectional cohorts [Males: IGFBP-2 mean iTRAQ log 2 ratio in high vs. Iow vitamin $D$ status $(S D)=0.6(0.8), p=0.02$; Females: IGFBP-3 mean iTRAQ $\log _{2}$ ratio in high vs. low vitamin $D$ status $(S D)=0.5(0.5)$, $p=0.001]$. b ELISA measurements confirmed the sexually dimorphic correlation of IGFBP-2 and IGFBP-3 with vitamin D status among adults with obesity [Males: IGFBP-2 Low vitamin D status median (25th to 75th percentile) $=5.1$ (2.9-11.1), High vitamin D status median (25th to 75th percentile) $=10.0$ (5.6-22.1), $p=0.002$; IGFBP-3 Low vitamin D status median (25th to 75th percentile) $=5.4$ (3.4-5.6), High vitamin D status median (25th to 75th percentile) $=5.5(3.9-5.8), p=0.66$ ] [Females: IGFBP-2 Low vitamin D status median (25th to 75th percentile) $=11.4(6.6-23.4)$, High vitamin $D$ status median (25th to 75 th percentile) $=13.4(5.7-25.8), p=0.99$; IGFBP-3 Low vitamin D status median (25th to 75 th percentile) $=2.4$ (1.6-2.8), High vitamin D status median (25th to 75th percentile) $=2.8(2.7-4.5) . p=0.03$ ]

from the kidney and liver ${ }^{30,31}$. Along these lines, patients with acromegaly exhibit increased gluconeogenic activity in the liver ${ }^{30}$ and are at risk of developing type 2 diabetes $^{32}$. Furthermore, studies have found that GH suppresses glucose uptake by the adipose tissue, through the down-regulation of glucose transporter substrates on the plasma membrane of adipocytes ${ }^{33}$.

However, the effects of GH on glycaemic control are complex, since GH stimulates the production of insulinlike growth factor I (IGF1). Thus, GH deficiency is paradoxically associated with insulin resistance and abdominal obesity, a phenomenon possibly attributed to decreased IGF1 activity ${ }^{34}$. IGF1, a hormone primarily produced by the liver, has growth-promoting properties and insulin-like effects that are exerted through binding with the IGF1 receptor and insulin receptor ${ }^{34,35}$. Insulinlike growth factor II (IGF2) is a hormone closely related to IGF1, that also exerts growth-regulating and insulinlike activities.
The IGF1 and IGF2 components are carried in the systemic circulation by the soluble insulin-like growth factor binding proteins (IGFBPs) ${ }^{36}$. There are six members in the IGFBP protein family (numbered 1 through 6) and their molecular weight varies from 24 to $45 \mathrm{kDa}^{37}$. The primary role of IGFBPs is to extend the half-life of IGFs in plasma ${ }^{38,55}$. However, studies have shown that IGFBPs can also inhibit the binding of IGF1 and IGF2 to their respective receptors ${ }^{39,40}$.

Vitamin D and IGFBPs have been suggested to act synergistically to affect insulin sensitivity, although the mechanism remains elusive ${ }^{40,41}$. IGFBPs have also been found to reflect risk for coronary heart disease and stroke as part of a randomised control trial using hormone therapy $^{42}$. A recent study demonstrated the relationships between IGF1 and its binding proteins, with cardiometabolic risk in hypertensive perimenopausal females ${ }^{43}$. Furthermore, it has been reported in patients with T2DM, that the IGF system is strongly associated with 


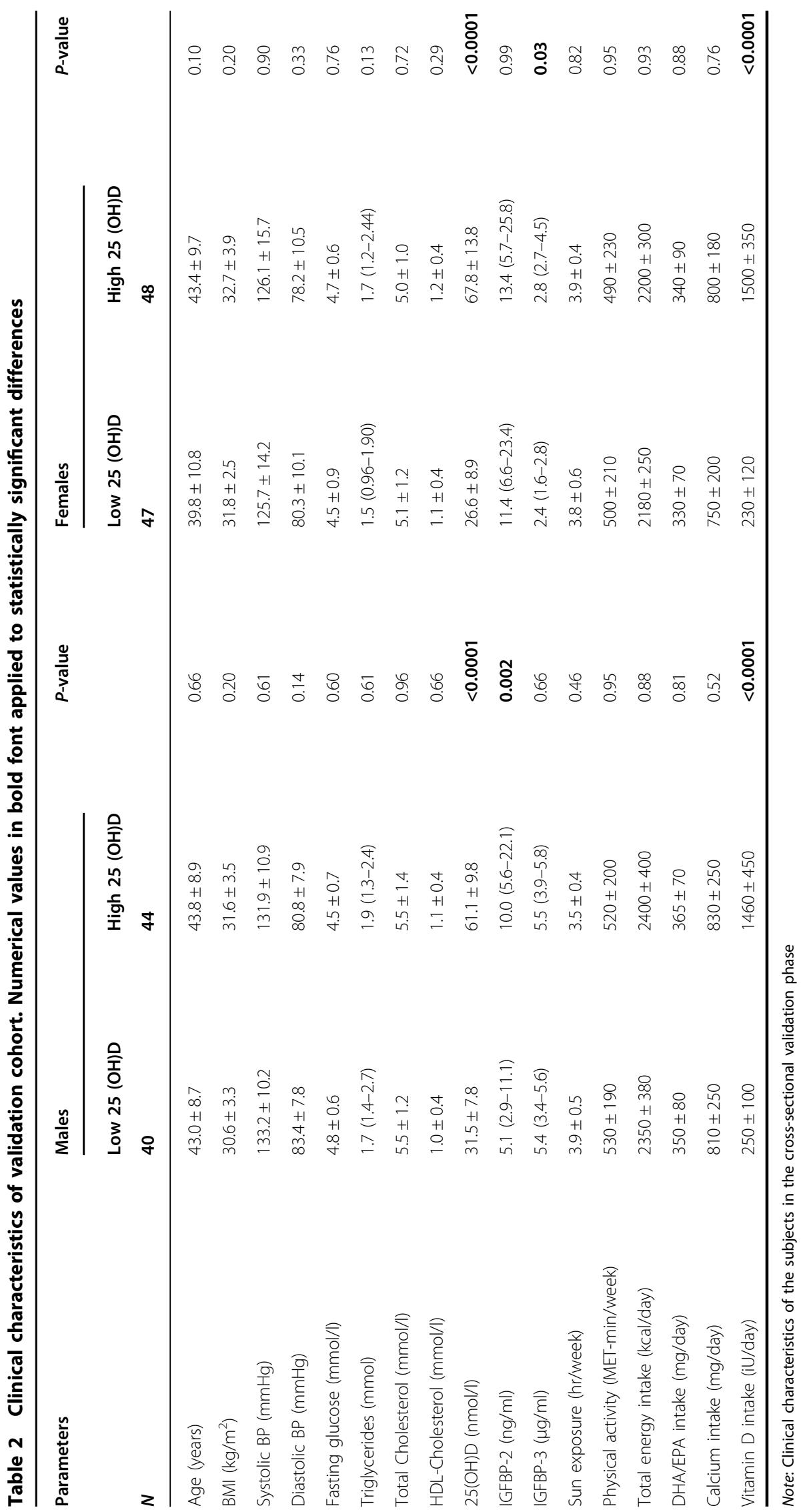


cardiovascular disease damage ${ }^{44}$ and may constitute alternative risk factor markers ${ }^{45}$. In this study, the nontargeted high-precision serum proteomics analysis of a cross-sectional discovery cohort with bioinformatics interpretation, literature research and biostatistical assessment, identified a novel sexually dimorphic correlation of IGFBP-2 and IGFBP-3 with vitamin D status in non-diabetic males and females with obesity (Fig. 4a). Additionally, the IGFBP-2 and IGFBP-3 proteomic findings were verified for relative quantitative accuracy with Luminex ELISA against a statistically significant number of samples from an independent cohort (Fig. 4b) but with analogous clinical conditions with the discovery cohort (Tables 1 and 2).

Although the precise function of IGFBP-2 in cardiometabolic pathophysiology is unclear, it has been implicated in the decrease of the biological activity of IGF1, thus regulating insulin sensitivity ${ }^{46}$. A study by Hedbacker et al. ${ }^{47}$ showed that the mRNA expression for IGFBP2 mediated the regulation of glucose metabolism, in response to the administration of physiologic and extraphysiologic doses of leptin in mice. In particular, high mRNA levels of IGFBP2 were associated with reduced blood glucose in wild type, as well as diabetic mice, and also suppressed hepatic glucose production and reduced expression of genes involved in hepatic fatty acid synthesis and gluconeogenesis. This study suggested that the induction of IGFBP2 as a result of leptin administration might play a preventive role in the pathogenesis of T2DM.

IGFBP-3 is the most prominent member of the IGFBP family, transporting $70-90 \%$ of the circulating IGF-1 and $-2^{39}$. Serum IGFBP-3 has been shown to increase as a result of vitamin $\mathrm{D}$ administration ${ }^{48}$. Vitamin $\mathrm{D}$ may increase IGFBP-3 levels either through direct transcriptional induction in the liver, since IGFBP-3 is a transcriptional target of the vitamin D receptor ${ }^{49}$, or through an indirect enhancement of growth hormone stimulation $^{50}$. IGFBP-3 decreases insulin-mediated uptake of glucose in the adipocytes ${ }^{51}$ and inhibits adipogenesis ${ }^{52}$.

We have previously shown that the serum proteomic profile of men is distinct from that of women, with men over-expressing proteins associated with an increased risk of cardiovascular disease ${ }^{25}$. Furthermore, sex hormones have been shown to affect circulating IGFBP levels ${ }^{53,54}$. Finally, we have described a sex-specific effect of vitamin D administration on serum proteins related to cardiovascular risk ${ }^{24}$. The above-mentioned associations could partly explain the results of the present study on a sexually dimorphic correlation of IGFBP-2 and IGFBP-3 with vitamin D status.

Despite the significant statistical power of this pilot study, the present findings should be examined in larger cross-sectional cohorts and randomised placebocontrolled studies to verify their translational importance. One study limitation is that some of the study participants were borderline hypertensive (systolic blood pressure up to $145 \mathrm{mmHg}$ ), an anticipated trend within a cohort of adults with overweight/obesity. Another study limitation is that serum IGF-1 levels were not measured in the present cohort. However, examining the association between IGF-1 levels and sex, vitamin D status and BMI constitutes a future perspective.

In conclusion, IGFBP-2 and IGFBP-3 were found to correlate with vitamin D status in males and female adults with obesity, respectively. In this population group, IGFBP-2 and IGFBP-3 warrant further examination as potential sexually dimorphic serological markers linking vitamin D status with cardiometabolic outcomes.

\section{Acknowledgements}

Funding: The Deanship of Scientific Research, Prince Mutaib Chair for Biomarkers of Osteoporosis in King Saud University, Riyadh, Saudi Arabia. JT was supported by the China Scholarship Council and the China Postdoctoral Science Foundation (2013T60260).

\section{Author details}

'Biochemistry Department, College of Science, Biomarkers Research Program, King Saud University, Riyadh, Saudi Arabia. ${ }^{2}$ Biochemistry Department, Prince Mutaib Chair for Biomarkers of Osteoporosis, King Saud University, Riyadh, Saudi Arabia. ${ }^{3}$ Centre for Proteomic Research, Institute for Life Sciences, University of Southampton, Southampton, UK. ${ }^{4}$ School of Pharmacy, Tianjin Medical University, Tianjin, China. ${ }^{5} 1$ st Department of Pediatrics, University of Athens, Athens, Greece. ${ }^{6}$ Cancer Sciences Unit, Faculty of Medicine, University of Southampton, Southampton, UK. 'Present address: Proteome Exploration Laboratory, Beckman Institute, Division of Biology and Biological Engineering, California Institute of Technology, Pasadena, CA 91125, USA

\section{Author contributions}

N.A.D., A.M., G.P.C., and S.D.G. concept and clinical design; A.M., D.G.B., M.F., J.T., and A.A. performed proteomics work; S.Y., Y.A.S., and S.S. performed verification work; A.M., D.G.B., and S.D.G. contributed new reagents and analytic tools; A.M., S.Y., S.S. and S.D.G. analyzed data; N.A.D., O.A.A., G.P.C., and S.D.G. supervised research; A.M. and S.D.G. writing-original draft; N.A.D., A.M., S.S., G.P.C. and S.D.G. writing-review and editing.

Conflict of interest

The authors declare that they have no conflict of interest.

\section{Publisher's note}

Springer Nature remains neutral with regard to jurisdictional claims in published maps and institutional affiliations.

Supplementary Information accompanies this paper at (https://doi.org/ 10.1038/s41387-018-0063-8).

Received: 27 May 2018 Revised: 20 August 2018 Accepted: 10 September 2018

Published online: 04 October 2018

\section{References}

1. Bikle, D. D. Vitamin D: an ancient hormone. Exp. Dermatol. 20, 7-13 (2011).

2. Reichrath, J., Zouboulis, C. C., Vogt, T. \& Holick, M. F. Targeting the vitamin D endocrine system (VDES) for the management of inflammatory and malignant skin diseases: an historical view and outlook. Rev. Endocr. Metab. Disord. 17, 405-417 (2016). 
3. Lehmann, U. et al. Vitamin D3 supplementation: response and predictors of vitamin D3 metabolites-A randomized controlled trial. Clin. Nutr. 35, 351-358 (2016).

4. Ross, A. C. et al. The2011 report on dietary reference intakes for calcium and vitamin D from the Institute of Medicine: what clinicians need to know. J. Clin. Endocrinol. Metab. 96, 53-58 (2011).

5. Holick, M. F. et al. Evaluation, treatment, and prevention of vitamin D deficiency: an Endocrine Society clinical practice guideline. J. Clin. Endocrinol. Metab. 96, 1911-1930 (2011).

6. Lee, J. P., Tansey, M., Jetton, J. G. \& Krasowski, M. D. Vitamin D toxicity: a 16-year retrospective study at an academic medical center. Lab. Med. 49, 123-129 (2018).

7. Kimball, S. M., Mirhosseini, N. \& Holick, M. F. Evaluation of vitamin D3 intakes up to 15,000 international units/day and serum 25 -hydroxyvitamin D concentrations up to $300 \mathrm{nmol} / \mathrm{L}$ on calcium metabolism in a community setting. Dermatoendocrino/ 9, e1300213 (2017).

8. Lips, P. Worldwide status of vitamin D nutrition. J. Steroid Biochem. Mol. Biol. 121, 297-300 (2010)

9. Cashman, K. D. et al. Vitamin D deficiency in Europe: pandemic? Am. J. Clin. Nutr. 103, 1033-1044 (2016).

10. Wang, L. et al. Circulating 25-hydroxy-vitamin D and risk of cardiovascular disease: a meta-analysis of prospective studies. Circ. Cardiovasc Qual. Outcomes 5, 819-829 (2012)

11. Garland, C. F. et al. Meta-analysis of all-cause mortality according to serum 25hydroxyvitamin D. Am. J. Public Health 104, e43-e50 (2014).

12. Zhang, R. et al. Serum 25-hydroxyvitamin D and the risk of cardiovascular disease: dose-response meta-analysis of prospective studies. Am. J. Clin. Nutr. 105, 810-819 (2017).

13. Lee, J. H., O'Keefe, J. H., Bell, D., Hensrud, D. D. \& Holick, M. F. Vitamin D deficiency an important, common, and easily treatable cardiovascular risk factor? J. Am. Coll. Cardiol. 52, 1949-1956 (2008).

14. Song, Y. et al. Blood 25-hydroxy vitamin D levels and incident type 2 diabetes: a meta-analysis of prospective studies. Diabetes Care 36, 1422-1428 (2013).

15. Pannu, P. K., Piers, L. S., Soares, M. J., Zhao, Y. \& Ansari, Z. Vitamin D status is inversely associated with markers of risk for type 2 diabetes: a population based study in Victoria, Australia. PLoS ONE 12, e0178825 (2017).

16. Lucato, P. et al. Low vitamin D levels increase the risk of type 2 diabetes in older adults: a systematic review and meta-analysis. Maturitas 100, 8-15 (2017).

17. Vangoitsenhoven, R. et al. Effect of a transcriptional inactive or absent vitamin $D$ receptor on beta-cell function and glucose homeostasis in mice. J. Steroid Biochem. Mol. Biol. 164, 309-317 (2016).

18. Garbossa, S. G. \& Folli, F. Vitamin D, sub-inflammation and insulin resistance. A window on a potential role for the interaction between bone and glucose metabolism. Rev. Endocr. Metab. Disord. 18, 243-258 (2017).

19. Muscogiuri, G. et al. Mechanisms in endocrinology: vitamin D as a potential contributor in endocrine health and disease. Eur. J. Endocrinol. 171, R101-R110 (2014).

20. Gulseth, H. L., Wium, C., Angel, K., Eriksen, E. F. \& Birkeland, K. I. Effects of vitamin d supplementation on insulin sensitivity and insulin secretion in subjects with type 2 diabetes and vitamin d deficiency: a randomized controlled trial. Diabetes Care 40, 872-878 (2017).

21. Lu, L. et al. Association of vitamin D with risk of type 2 diabetes: a Mendelian randomisation study in European and Chinese adults. PLoS. Med. 15, e1002566 (2018).

22. von Elm, E. et al. The Strengthening the Reporting of Observational Studies in Epidemiology (STROBE) statement: guidelines for reporting observational studies. Lancet 370, 1453-1457 (2007).

23. Al-Daghri, N. M. et al. Diabetes mellitus type 2 and other chronic noncommunicable diseases in the central region, Saudi Arabia (Riyadh cohort 2): a decade of an epidemic. Bmc. Med. 9, 76 (2011).

24. Al-Daghri, N. M. et al. Sex-specific vitamin D effects on blood coagulation among overweight adults. Eur. J. Clin. Invest. 46, 1031-1040 (2016).

25. Al-Daghri, N. M. et al. Whole serum 3D LC-nESI-FTMS quantitative proteomics reveals sexual dimorphism in the milieu interieur of overweight and obese adults. J. Proteome Res. 13, 5094-5105 (2014).

26. Levin, $Y$. The role of statistical power analysis in quantitative proteomics. Proteomics 11, 2565-2567 (2011).

27. Concato, J., Peduzzi, P., Holford, T. R. \& Feinstein, A. R. Importance of events per independent variable in proportional hazards analysis. I. Background, goals, and general strategy. J. Clin. Epidemiol. 48, 1495-1501 (1995).
28. Le Roith, D. Seminars in medicine of the Beth Israel Deaconess Medical Center. Insulin-like growth factors. N. Engl. J. Med. 336, 633-640 (1997).

29. Møller, N. \& Jørgensen, J. O. Effects of growth hormone on glucose, lipid, and protein metabolism in human subjects. Endocr. Rev. 30, 152-177 (2009).

30. Schwarz, J. M. et al. Effects of recombinant human growth hormone on hepatic lipidand carbohydrate metabolism in HIV-infected patients with fataccumulation. J. Clin. Endocrinol. Metab. 87, 942 (2002).

31. Höybye, C. et al. Contribution of gluconeogenesis and glycogenolysis to hepatic glucose production in acromegaly before and after pituitary microsurgery. Horm. Metab. Res. 40, 498-501 (2008).

32. Vijayakumar, A., Yakar, S. \& Leroith, D. The intricate role of growth hormone in metabolism. Front Endocrinol. (Lausanne). 2, 32 (2011).

33. Kilgour, E., Baldwin, S. A. \& Flint, D. J. Divergent regulation of rat adipocyte GLUT1 and GLUT4 glucose transporters by GH. J. Endocrinol. 145, 27-33 (1995).

34. Kim, S. H. \& Park, M. J. Effects of growth hormone on glucose metabolism and insulin resistance in human. Ann. Pediatr. Endocrinol. Metab. 22, 145-152 (2017).

35. Anisimov, V. N. \& Bartke, A. The key role of growth hormone-insulin-IGF-1 signaling in aging and cancer. Crit. Rev. Oncol. Hematol. 87, 201-223 (2013).

36. Shimasaki, S., Shimonaka, M., Zhang, H. P. \& Ling, N. Identification of five different insulin-like growth factor binding proteins (IGFBPs) from adult rat serum and molecular cloning of a novel IGFBP-5 in rat and human. J. Biol. Chem. 266, 10646-10653 (1991).

37. Hwa, V., Oh, Y. \& Rosenfeld, R. G. The insulin-like growth factor-binding protein (IGFBP) superfamily. Endocr. Rev. 20, 761-787 (1999).

38. Clemmons, D. R. et al. Role of insulin-like growth factor binding proteins in the control of IGF actions. Prog. Growth Factor Res. 6, 357-366 (1995).

39. Wetterau, L. A., Moore, M. G., Lee, K. W., Shim, M. L. \& Cohen, P. Novel aspects of the insulin-like growth factor binding proteins. Mol. Genet. Metab. 68 161-181 (1999).

40. Forouhi, N. G., Luan, J., Cooper, A., Boucher, B. J. \& Wareham, N. J. Baseline serum 25-hydroxy vitamin d is predictive of future glycemic status and insulin resistance: the Medical Research Council Ely Prospective Study 1990-2000. Diabetes 57, 2619-2625 (2008).

41. Kamycheva, E., Berg, V. \& Jorde, R. Insulin-like growth factor I, growth hormone, and insulin sensitivity: the effects of a one-year cholecalciferol supplementation in middle-aged overweight and obese subjects. Endocrine 43, 412-418 (2013).

42. Prentice, R. L. et al. Proteomic risk markers for coronary heart disease and stroke: validation and mediation of randomized trial hormone therapy effects on these diseases. Genome Med. 5, 112 (2013).

43. Olszanecka, A., Dragan, A., Kawecka-Jaszcz, K., Fedak, D. \& Czarnecka, D. Relationships of insulin-like growth factor-1, its binding proteins, and cardiometabolic risk in hypertensive perimenopausal women. Metabolism 69, 96-106 (2017).

44. Hjortebjerg, R. et al. The IGF system in patients with type 2 diabetes: associations with markers of cardiovascular target organ damage. Eur. J. Endocrinol. 176, 521-531 (2017).

45. Kaplan, R. C. et al. Insulinlike growth factor binding protein-1 and ghrelin predict health outcomes among older adults: cardiovascular health study cohort. J. Clin. Endocrinol. Metab. 102, 267-278 (2017).

46. Arafat, A. M. et al. The role of insulin-like growth factor (IGF) binding protein-2 in the insulin-mediated decrease in IGF-I bioactivity. J. Clin. Endocrinol. Metab. 94, 5093-5101 (2009)

47. Hedbacker, K. et al. Antidiabetic effects of IGFBP2, a leptin-regulated gene. Cell. Metab. 11, 11-22 (2010).

48. Bereket, A. et al. Circulating insulin-like growth factor binding protein-4 (IGFBP4 ) is not regulated by parathyroid hormone and vitamin D in vivo: evidence from children with rickets. J. Clin. Res. Pediatr. Endocrinol. 2, 17-20 (2010).

49. Krishnan, A. V. et al. Analysis of vitamin D-regulated gene expression in LNCaP human prostate cancer cells using cDNA microarrays. Prostate 59, 243-251 (2004).

50. Liao, L., Chen, X., Wang, S., Parlow, A. F. \& Xu, J. Steroid receptor coactivator 3 maintains circulating insulin-like growth factor I (IGF-I) by controlling IGFbinding protein 3 expression. Mol. Cell. Biol. 28, 2460-2469 (2008).

51. Chan, S. S., Twigg, S. M., Firth, S. M. \& Baxter, R. C. Insulin-like growth factor binding protein-3 leads to insulin resistance in adipocytes. J. Clin. Endocrinol. Metab. 90, 6588-6595 (2005) 
52. Kong, J. \& Li, Y. C. Molecular mechanism of 1,25-dihydroxyvitamin D3 inhibition of adipogenesis in 3T3-L1 cells. Am. J. Physiol. Endocrinol. Metab. 290, E916-E924 (2006).

53. Münzer, T. et al. Effects of GH and/or sex steroids on circulating IGF-I and IGFBPs in healthy, aged women and men. Am. J. Physiol. Endocrinol. Metab. 290, E1006-E1013 (2006).
54. Janssen, J. A. et al. Serum free IGF-I, total IGF-I, IGFBP-1 and IGFBP-3 levels in an elderly population: relation to age and sex steroid levels. Clin. Endocrinol. 48, 471-478 (1998).

55. Wenjing Ruan, Maode Lai, (2010) Insulin-like growth factor binding protein: a possible marker for the metabolic syndrome?. Acta Diabetologica 47: $5-14$ 